\title{
Towards a Commitment-based Reference Ontology for Services
}

\author{
Julio Cesar Nardi ${ }^{1,2}$, Ricardo de Almeida Falbo², João Paulo A. Almeida ${ }^{2}$, Giancarlo Guizzardi², \\ Luís Ferreira Pires ${ }^{3}$, Marten J. van Sinderen ${ }^{3}$, Nicola Guarino ${ }^{4}$ \\ ${ }^{1}$ Informatics Department, Federal Institute of Espírito Santo, Colatina, Brazil \\ ${ }^{2}$ Ontology \& Conceptual Modeling Research Group (NEMO), Federal University of Espírito Santo, Vitória, Brazil \\ ${ }^{3}$ Centre for Telematics and Information Technology, University of Twente, Enschede, The Netherlands \\ ${ }^{4}$ Laboratory for Applied Ontology, Institute of Cognitive Sciences and Technologies, Trento, Italy \\ julionardi@ifes.edu.br, \{falbo,jpalmeida, gguizzardi\}@inf.ufes.br, \\ \{1.ferreirapires, m.j.vansinderen\}@utwente.nl,nicola.guarino@cnr.it
}

\begin{abstract}
The concept of "service" has been characterized by different disciplines and authors from various points of view. The variety of characterizations reveals that this notion, although an intuitive one, is far from trivial. Given the importance of services in enterprise computing and Service Science in general, we believe that a clear account of services and service-related concepts is necessary and would serve as a basis for communication, consensus and alignment of various approaches and perspectives. In this paper we propose a commitment-based account of the notion of service captured in a core reference ontology called UFO-S. We address the commitments established between service providers and customers, and show how such commitments affect the service lifecycle. We show that the commitment-based account can serve to harmonize different notions of service in the literature.
\end{abstract}

Keywords: service science, service computing, core ontology.

\section{INTRODUCTION}

The notion of "service" has had a major impact on Marketing, Operations and Computer Science over the last decades, leading to cross-discipline efforts under the banners of "Service Science" and "Service Computing". Several authors from different disciplines have attempted to define this notion under various perspectives, leading to a variety of characterizations for "services", each emphasizing different aspects. For example, the service as interaction perspective focuses on the interactions among service participants to achieve results or generate effects [1]; service as value cocreation focuses on services as the basis of economic exchange [2][3][4]; service as capability focuses on the capability of a provider to produce benefits to customers (e.g., [5][6]); service as application of competences focuses on the manifestation of the capability of one party to act in benefit of another party [2]; finally, service as software focuses on pieces of software that can be accessed through well-defined interfaces [7].

We believe these characterizations are influenced, among others, by many aspects, such as the research community to which their authors belong (e.g., Service Science, or Service Computing), the targeted application area (e.g., Data Communication, or Enterprise Architecture), or the layer in an Enterprise Architecture (Business, Application, and Technology [8]). The variety of characterizations for the notion of service reveals that it is far from trivial. As a consequence, under close inspection, we can conclude that the term "service" often denotes different (possibly related) underlying concepts. As discussed by Alter in [9], "even if different communities of practice can live with their own somewhat inconsistent views of service, conflicting views of service surely cannot facilitate effective communication between business and IT practitioners and between business and computer science researchers".

Given the importance of services in Services Computing and Service Science in general, we believe that a clear account the underlying concepts is necessary and would serve as a basis for communication, consensus and alignment of the various approaches and perspectives. Thus, the account we propose in this paper is intended to address the notion of service broadly, with application to both Service Science and Service Computing. We approach this account by proposing a core reference ontology for services called UFO-S.

As a core ontology [10][11], UFO-S refines concepts of a foundational ontology, by providing a conceptualization for services that is independent of a particular application domain. The foundational ontology we adopt is the Unified Foundational Ontology (UFO) [12][13], which provides us with basic concepts for objects and events, their types, relations and properties, as well as intentional and social elements to account for agents, the social relations they establish, the actions they undertake, etc. By grounding UFO-S in a foundational ontology we are able to reveal important conceptual distinctions that would otherwise be ignored in informal characterizations of services.

Our account builds upon earlier works that treat services under the perspective of the commitments involved in service relations [14][15][16][17]. This perspective emphasizes that throughout the service life-cycle, commitments of several natures are established between service providers and service customers. We address three main aspects: (i) the characterization of commitments (and corresponding claims) in service relationships; (ii) the roles played by agents in service relationships, as a consequence of the established commitments; and (iii) the dynamics of the relationships between the agents along the service life-cycle, in which commitments are established and fulfilled.

This paper is further structured as follows: Section II motivates the commitment-based characterization of services; Section III presents the foundational ontology we adopt as a basis for UFO-S; Section IV presents UFO-S; Section V evaluates UFO-S by showing how it explains and relates various service perspectives; Section VI discusses related work; and, Section VII presents our conclusions. 


\section{SERVICE AND COMMITMENTS}

A number of works in Service Science [9][15][17] and Service Computing [18][19][20] explicitly mention commitments/promises/obligations established between service participants in the service life-cycle. For Dumas et al. [20], a service instance is essentially a promise by one party (the provider), to perform a function on behalf of another party (the consumer). Ferrario and Guarino [15] associate the concept of service to an explicit commitment between provider and customer, and also to the corresponding service process. Santos et al. [18] define service as a temporal entity related to the commitment (the service agreement) of the provider in performing a type of action on behalf of a client whose outcome satisfies a client's goal. Sullivan [19] defines the notion of "obligation" for capturing the responsibilities of both the service provider and the service requester.

The benefits of a characterization based on commitments have been discussed from the perspective of business [17] as well as IT [16]. For example, Alter [9] points out that the balance of the (co)responsibilities, associated to the importance of commitments for governing service delivery, are important aspects for characterizing service dynamics. Mingming and Youbei [17] highlight that service commitments are useful to deal with service intangibility, by offering means to convert intangible service aspects into more "concrete service standards". Singh and colleagues [16] remark that commitments can be used for raising the low-level of abstraction of existing service-oriented architectures, allowing to reduce the gap between the business and the IT perspectives. In their view, commitments capture business meaning, which is not directly represented in process-oriented approaches such as BPMN [16].

Further, some kinds of service business models cannot be suitably explained without the notion of commitments. Ferrario and Guarino [15] explain that by using as an example an insurance service, in which the customer pays for having someone (an insurer) who is committed to intervene in case of an accident. Arguably, the customer hopes that the actions of the service process are never to be performed. In this case, the service is provided by the existence of a commitment even if no actions are executed.

Commitments affect the overall life-cycle of services including (i) service offer, (ii) service negotiation, and (iii) service delivery. Service offer is the initial phase in which services are presented to target customers, and service aspects such as provider availability, pricing, payment, security, quality of service, and reputation [20] are described and published. Service negotiation is characterized by the interaction between customer and provider in order to establish an agreement about their responsibilities [15]. If service negotiation is successfully achieved, a service agreement is established, determining what has been settled between service participants for service delivery. Finally, service delivery concerns the execution of actions to fulfill the established commitments [20]. The broad impact of commitments in the service life-cycle, as well as the benefits of such a view, motivate us to consider a commitment-based account as a focal point for our core ontology for services.

\section{THE UNIFIED FOUNDATIONAL ONTOLOGY}

Since it is defined as a core ontology, UFO-S is situated in a region between foundational and domain ontologies. A core ontology provides a refinement to a foundational ontology in a specific field. In this sense, foundational ontologies serve as basis for core ontologies, as core ontologies do for domain ontologies [11]. In accordance with this view, UFO-S was designed taking as basis the Unified Foundational Ontology (UFO).

UFO is a foundational ontology developed with an interdisciplinary approach inspired by Formal Ontology, Philosophical Logic, Linguistics, and Cognitive Psychology. It is based on DOLCE [21], OntoClean [22], and GFO/GOL [23]. It consists of three main parts: UFO-A, an ontology of endurants (objects) [12], UFO-B, an ontology of events (perdurants) [13], and UFO-C [13][24], an ontology of social entities built on the top of UFO-A and UFO-B.

Our choice of using UFO can be justified by successful application of this foundational ontology in previous works to evaluate, redesign, and ground ontologies, models, and frameworks of several research areas, such as Software Engineering, Conceptual Modeling, and Enterprise Modeling $[13][25][26][27][28][29]$. The UFO fragments that are relevant to this work are presented below.

\section{A. UFO-A and UFO-B}

The fundamental distinction in UFO [12] is between individuals and universals. Individuals are entities that exist in reality and possess a unique identity, while universals are patterns of features that can be realized in a number of different individuals. UFO-A is an ontology of endurants whose patterns of features are called endurant universals [12]. UFO-B, in turn, is an ontology of events (perdurants) whose patterns of features are event universals [13].

Fig. 1 presents a fragment of $\operatorname{UFO}(\mathrm{A}$ and $\mathrm{B})$ that focuses on categories of individuals.

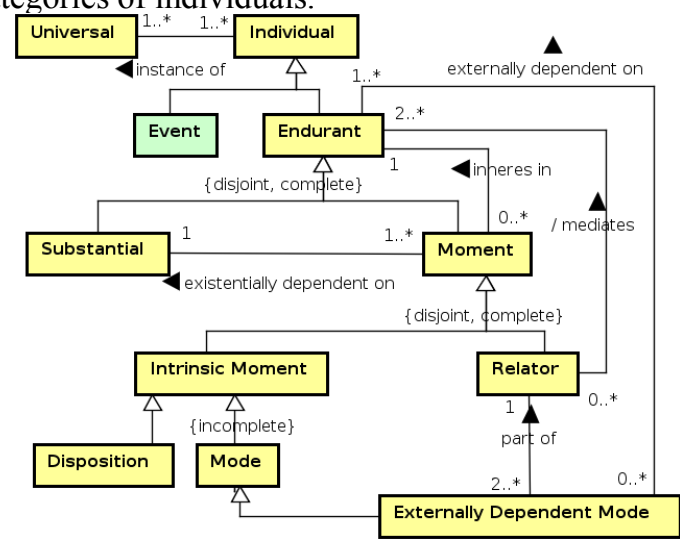

Figure 1. A fragment of UFO-A and UFO-B (Individuals).

Endurants are individuals that are wholly present whenever they are present, and can be divided into substantials and moments. Substantials are existentially independent endurants (e.g., a person, a car). Moments are individuals that can only exist in other individuals, and, thus, they are existentially dependent on their bearers (e.g., a person's headache, a covalent bond between atoms) [12]. 
Intrinsic moments are dependent on one single individual (e.g., an apple's color). Modes are intrinsic moments that are not directly measurable (e.g., John's desires, intentions, perceptions, symptoms, skills) [12]. Following [30][31], dispositions in UFO are intrinsic moments (e.g., the fragility of a glass) that are manifested in particular situations through the occurrence of certain events, and that can also fail to be manifested. Take for example the disposition of a magnet to attract metallic material. The magnet has this disposition even if it is never manifested, e.g., because the magnet was never close to any magnetic material. Nonetheless, it can certainly be said to possess that intrinsic property.

Relators are moments that existentially depend on two or more endurants (e.g., an employment, an enrollment, a medical treatment, a marriage) [12]. For example, consider that John and Mary are married. In this case, the relator (their marriage) aggregates all externally-dependent modes that they acquire by virtue of participating in this relation (e.g, all commitments and claims towards each other) and that share the same foundational event (in this case the wedding event). In our example, John bears responsibilities and rights towards Mary. As counterpart, Mary bears the properties she acquires by being married with John.

Fig. 2 presents a fragment of UFO (A and B) that focuses on the categories of universals.

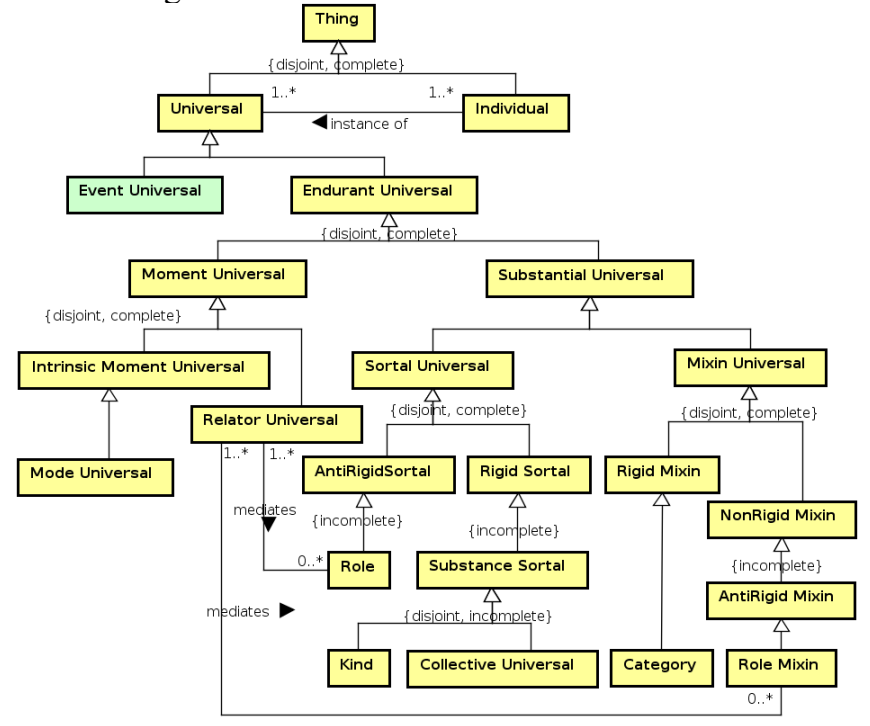

Figure 2. A fragment of UFO-A and UFO-B (Universals).

In UFO-A, substantial universal and moment universal are kinds of universals whose individuals are substantial individuals and moments [12], respectively. Concerning the substantial universal hierarchy, Sortal universals are substantial universals that carry a principle of identity for their individuals (e.g., Apple, Person, Student). The specialization of Sortal Universal is based on a metaproperty called rigidity. A universal is rigid if it necessarily applies to its instances, i.e., in every possible world (e.g., Apple, Person). Kinds are rigid sortal universals that provide a principle of identity for substantial individuals that instantiate them (e.g., Person). Collective Universals are rigid universals that represent collections of individuals with uniform structure (e.g., deck of cards, a forest, a group of people, a pile of bricks). This universal provides a principle of identity for the instances of that collection (but not for every individual in the collection), which can be intensional or extensional [12]. In contrast to rigid universals, a universal is anti-rigid if it does not apply necessarily to all its instances. For example, an individual $x$, which is an instance of the universal Student in a world $w 1$ can cease to instantiate this universal in another world $w 2$ without ceasing to exist as the same individual (i.e., as the same Person). Roles are antirigid and relationally-dependent sortal universals (e.g., Student) [12]. This means that roles are played by an object whenever there is a relator connecting it to other objects.

Mixin Universals are substantial universals that represent an abstraction of properties that are common to multiple disjoint kinds and, therefore, do not carry a unique principle of identity for their instances (e.g., Physical Object, Living Entity). Category represents a rigid and relationallyindependent mixin universal that aggregates essential properties that are common to different kinds (e.g., Intelligent Agent). Role Mixin, in turn, represents an antirigid and externally-dependent non-sortal universal, which aggregates properties that are common to different roles.

Finally, as opposed to endurants, events are individuals composed of temporal parts. They happen in time in the sense that they extend in time and accumulate temporal parts (e.g., a conversation, a business process). Whenever an event is present, it is not the case that all its temporal parts are present. Event universals are patterns of features that can be realized in a number of different events [13]. Events can be complex or atomic. Atomic events have no proper parts. Complex events are aggregations of at least two disjoint events [13]. The notion of foundation as a type of historical dependence [32][33] offers an important grounding for associating events to relators, since relators are founded by events. For example, the marriage relator is founded on a particular wedding event [32]. In this work, this notion is important because it offers means to explain the association between events in a service life-cycle, and the relators created among service participants, as discussed in Section IV.

\section{B. $\mathrm{UFO}-\mathrm{C}$}

$\mathrm{UFO}-\mathrm{C}$ is an ontology of social entities that specializes UFO-A and UFO-B [13][24]. A basic distinction in UFO-C is related to agents and (non-agentive) objects. Agents are agentive substantial individuals that are classified as physical agents (e.g., a person) or social agents (e.g., an organization, a society). Objects are non-agentive substantial individuals that are classified in physical objects (e.g., a book, a table) and social objects (e.g., money, language). A normative description is a type of social object that defines one or more rules/norms recognized by at least one social agent, and that can define nominal universals, such as social moment universals (e.g., social commitment types), social objects (e.g., the crown of the King of Spain) and social roles (e.g., president, or pedestrian). Examples of normative descriptions include contracts in general, but also sets of directives on how to perform actions within an organization. A plan 
description is a special type of normative description that describes complex action universals (complex plans) [25] (e.g., process, and guidelines). Fig. 3 presents a fragment of UFO-C that focuses on the distinction between agents and objects, and on the definition of normative description.

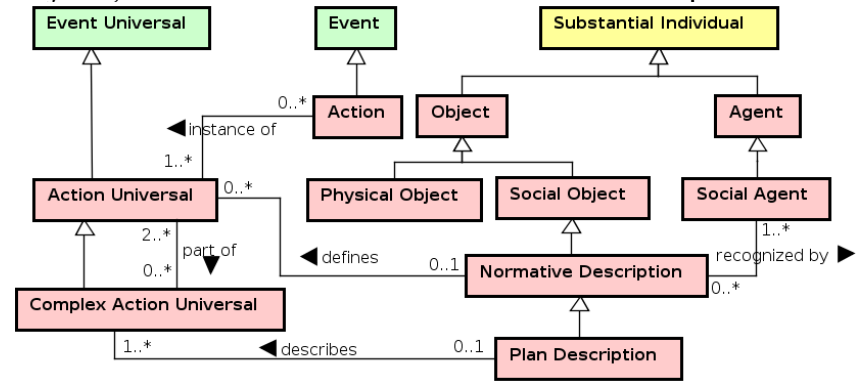

Figure 3. Agents, Objects, and Normative Description.

Figure 4 presents a fragment of UFO-C that focuses on types of intentional moments. Agents are substantials that can bear special types of modes named intentional moments. In this case, intentionality refers to the capacity of some properties of certain individuals to refer to possible situations in reality. Thus, intentional moments have a propositional content (proposition), which is an abstract representation of a class of situations referred by that intentional moment.

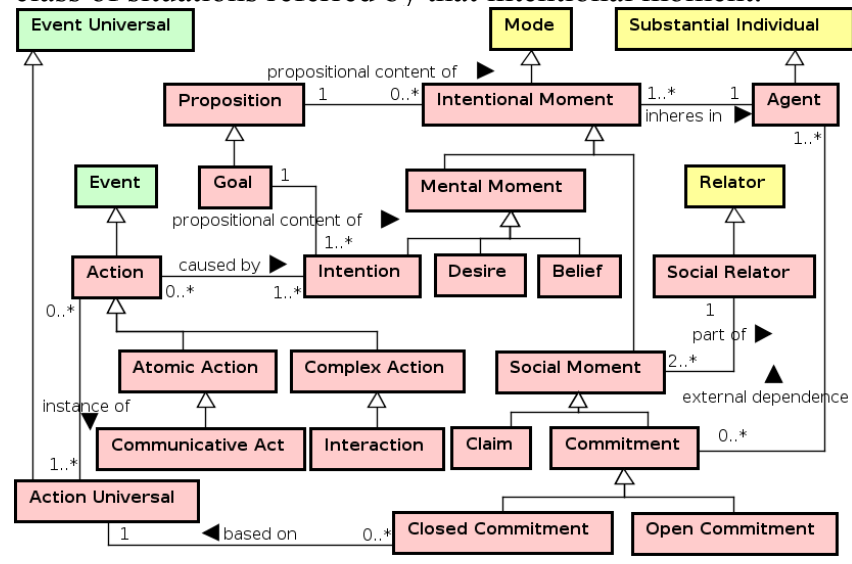

Figure 4. Actions, mental moments and social moments.

Intentional moments inhere in agents and can be mental or social. Mental moments are specialized in intentions (internal commitments), beliefs, and desires. Belief can be justified by situations in reality (e.g., my belief that the Moon orbits the Earth). Desires and intentions can be fulfilled or frustrated. Whilst a desire expresses a will of an agent towards a state of affairs in reality, intentions are desired state of affairs for which the agent commits to pursuing [34][35]. Intentions cause the agent to perform actions. Actions are types of events that can be complex or atomic. Communicative acts (a speech act such as inform, ask or promise in the sense of [34]) are types of atomic actions [13]. Interactions are types of complex actions composed of action contributions from different agents.

Social moments are types of intentional moments that are created by social actions (e.g., an interaction composed of the exchange of communicative acts). Social commitments and social claims are types of social moments. Social relators are relators composed of one or more pairs of social commitments and social claims [25]. As with all relators, social relators are founded in particular events. What "counts as" the founding event of a social relator, as well as the social responsibility and claims entailed by that social relator, depends on a normative description valid in that social context. For example, a particular constitution can define the responsibility and claims entailed by the marriage relator type, and that a particular speech act (e.g., "I hereby declare you husband and wife") uttered by a Judge in a specific context (e.g., in the presence of witnesses) is sufficient for the creation of a relator of that type.

As an additional example, suppose that John rents a car at a car rental office. When signing a business contract, John performs a communicative act (a promise). This act creates a commitment (a social commitment towards that organization) to return the car in a certain state. It also creates a social claim of the rental car office towards John with respect to that particular propositional content. Commitments and claims always form a pair that refers to a unique propositional content. Commitments are classified in open and closed. In an open commitment, the agents responsible for fulfilling the commitment are free to define how they will do this. In a closed commitment, the agent must fulfill the commitment by performing an action that is an instance of a plan defined by another agent [24].

\section{A COMmitMENT-BASEd SERVICE ONTOLOGY}

UFO-S is a commitment-based service ontology whose conceptualization is based on the establishment and fulfillment of commitments and claims between service participants (used to refer to both service provider and service customer) along the service life-cycle. In this paper, we focus on the three main phases of the service life-cycle, namely: (i) service offer, when a service is presented and made available to a target customer community, (ii) service negotiation, when provider and customer(s) negotiate in order to establish an agreement about specific aspects that drive the service delivery, and (iii) service delivery, when actions are performed in order to fulfill a service agreement. Despite their relevance to certain areas of investigation (such as services marketing), we consider all service-related actions that occur before service offer and after service delivery as outside the scope of this paper.

In order to represent UFO-S, we employ OntoUML [12], an UML profile that incorporate the foundational distinctions in UFO-A and UFO-B. Table I presents the OntoUML stereotypes used in this paper.

TABLE I. A SubSET OF ONTOUML STEREOTYPES

\begin{tabular}{|l|l|}
\hline \multicolumn{1}{|c|}{ Stereotype } & \multicolumn{1}{c|}{ Corresponding Concept in UFO } \\
\hline$<<$ category $>>$ & Category \\
\hline$<<$ kind $>>$ & Kind \\
\hline$<<$ collective $>>$ & Collective Universal \\
\hline$<<$ rolemixin $>>$ & Role Mixin \\
\hline$<<$ role $>>$ & Role \\
\hline$<<$ mode $>>$ & Mode Universal \\
\hline$<<$ relator $>>$ & Relator Universal \\
\hline$<<$ event $>>$ & Event Universal \\
\hline
\end{tabular}


In addition to employing OntoUML to represent UFO-S, we further ground UFO-S with respect to the social and intentional concepts of UFO-C.

\section{A. Service Offer}

Figure 5 shows an OntoUML class diagram with the main concepts and relations involved in a service offer, according to UFO-S. A service offer event results in the establishment of a service offering between a service provider and a target customer community. A service offering is composed of service offering commitments from the service provider towards the target customer community and the corresponding service offering claims from the target community towards the service provider.

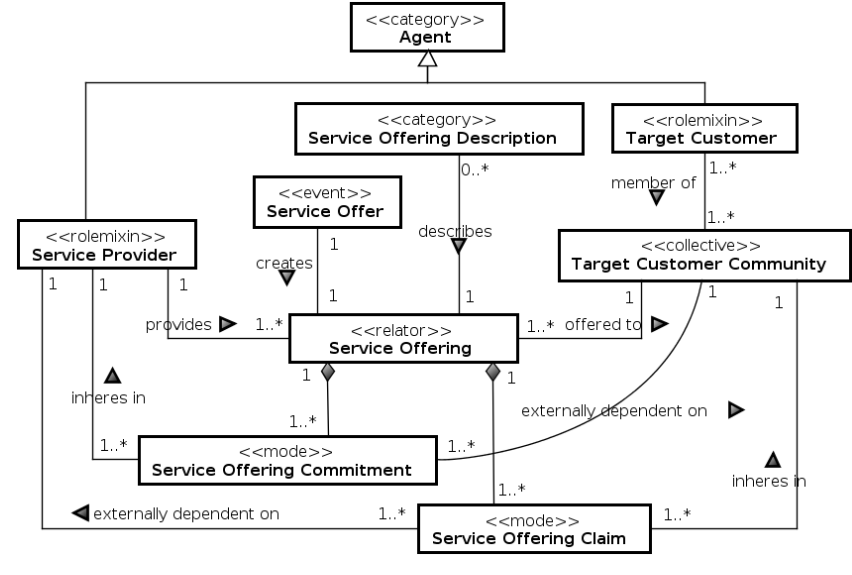

Figure 5. Service Offer

What "counts as" a service offer (i.e., which actions are service offers) depends ultimately on the (social) context in which services are offered; a service offer could thus be the registration of a service provider organization in a chamber of commerce, service advertisements, face-to-face communication, etc. The context will also determine the kinds of commitments that are established and the consequences that arise from a failure to fulfill such commitments. For example, in some legal systems, it is unlawful for an organization that has offered a service to refuse arbitrarily to provide the service to a particular customer in case no legitimate business reason is provided (in order to rule out arbitrary discrimination).

The set of statements (content) associated to the offering commitments (and corresponding claims) depends on the particular service business model, and, therefore, can refer to several different elements, such as conditions and requirements for providing the service, types of actions to be performed in the scope of service delivery, constraints, required customer's commitments (such as payment), etc. Such elements may be described in service offering descriptions (such as folders, registration documents in a chamber of commerce, artifacts in a service registry, etc.).

Take as example the case of a car rental service. When this service is offered by a particular car rental office called Highway (e.g., through advertisements), the car rental office plays the role of a service provider. It commits, under certain conditions, to grant temporary use of a vehicle to the customer. Examples of such conditions include minimum period of rental, car availability, qualifications and properties of the renter (e.g., should be a registered driver older than 21), expected payment guarantees, minimal rental period, etc. The members of the target community are entitled to rent a car if all conditions are fulfilled.

What is established in a service offering also determines the level of flexibility for a subsequent service negotiation phase, in which a particular service customer and a service provider establish a particular service agreement. Because of that, offering commitments are in fact meta-commitments [35] (i.e., they are commitments to accept commitments), because they refer to commitments that can be established later during the negotiation phase and that do not yet exist as a result of a service offer alone.

Agent is a category that represents the essential properties of any type of agentive substantial, such as person, organization, or software agent, which may have distinct principles of identity. Service provider is the role played by agents when these agents commit themselves to a target customer community by a set of offering commitments. In terms of UFO, service provider is a role mixin, since it can be instantiated by agents of different kinds, e.g., persons and organizations. Target customer community is a collective that refers to the group of agents that constitute the community to which the service is being offered. The community has an intensional principle of identity, in the sense that agents can enter or leave the community without altering the community's identity. The intensional criteria for defining the target customer community are included in the content of the service offering. This may range from offerings with no restrictions to strictly targeted service offerings.

The target customer is the role played by agents when they become members of a target customer community and, as consequence, have claims for the fulfillment of the commitments established by the agent playing the role of service provider. A service offering is the social relator that arises from the service offer event, and that can be described in service offering descriptions, i.e., normative descriptions in UFO-C. A service offering is the aggregate of offering commitments and the corresponding claims. Service offering commitments and claims are social moments (in the sense of UFO-C), i.e., offering commitments are intrinsic moments, which inhere in the meta-committed agent (acting as service provider) and are externally dependent on the target customer community. Offering claims, in turn, are intrinsic moments that inhere in the target customer community and are externally dependent on the meta-committed agent (acting as service provider).

\section{B. Service Negotiation}

Figure 6 shows an OntoUML class diagram with the main concepts and relations involved in service negotiation, according to UFO-S. Once a service is offered, service negotiation may occur. In general, service negotiation is motivated by the interest of a target customer in the service offering, considering its contents (including the conditions to be satisfied by the service customer in the case it should hire the service). During service negotiation, service provider and target customer interact in order to establish an agreement 
regarding their commitments and claims with respect to an eventual service delivery.

If service negotiation succeeds, a service agreement is established, and the service provider starts to play the role of hired service provider, while the target customer starts to play the role of service customer. Like a service offering, a service agreement is composed of commitments and claims. However, differently from the service offering, in a service agreement service customers may also establish commitments to service providers (e.g., the commitment to pay). Service agreement involves not only commitments from the hired service provider towards the service customer, but may also involve commitments from the service customer towards the hired service provider. Thus, these two participants become co-responsible for the service delivery.

Service agreement should conform to what was previously established in the corresponding service offering. This is exactly the relation between the meta-commitments in the offering and the commitments in the agreement. An agreement $X$ conforms to an offering $Y$ if for every metacommitment $m c$ in $Y$, there is a commitment $c$ in $X$ such that: any situation which satisfies the propositional content of $c$ satisfies the propositional content of $m c$ (and mutatis mutandis for claims).

As in the case of service offer, what is agreed between the parties (commitments and claims of both hired provider and service customer) depends on the context of the service agreement, as well as on the particular service business model, and, therefore, can refer to several different elements. These elements may be described in service agreement descriptions (such as a service contract)

Returning to the case of the car rental service, when John, a particular target customer, goes to the Highway car rental office and rents a car, he becomes a service customer, whereas Highway acts as a hired service provider. John and Highway commit themselves to perform some actions and to respect certain conditions. Examples of these conditions include amount to be paid per day, period of rental, conditions of the vehicle and so on. These conditions are registered in a contract.

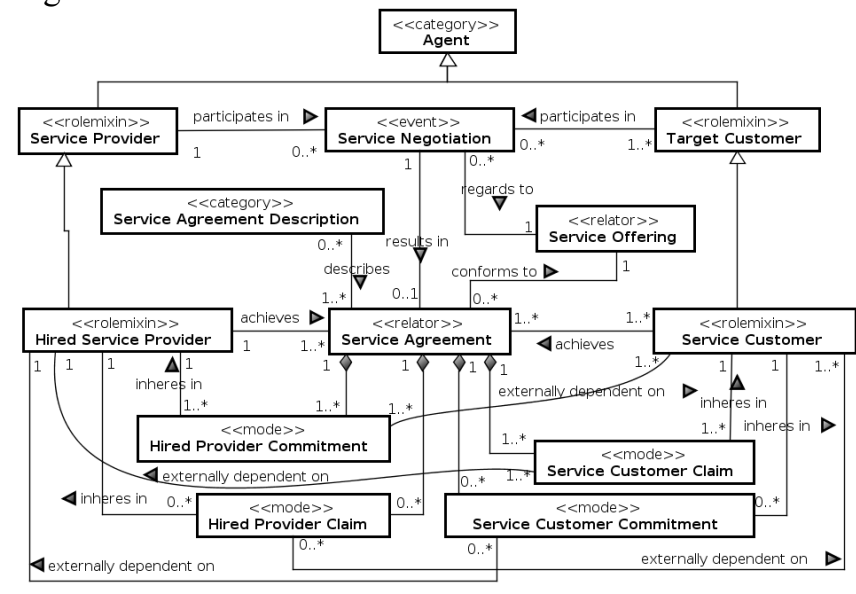

Figure 6. Service Negotiation

In terms of UFO-C, service negotiation is an interaction involving the participations of the service provider and the target customers. When service negotiation (an event) succeeds, this event is the foundation for a service agreement (a relator). Hired provider and service customer commitments and claims are social moments. Hired provider commitments and claims are intrinsic moments that inhere in a hired service provider and are externally dependent on a service customer. Service customer commitments and claims are intrinsic moments that inhere in a service customer and are externally dependent on a hired service provider.

In a manner analogous to how a service offering (as a social relator) mediates the relation between service provider and target service customers by aggregating offering commitments and claims, a service agreement mediates the relation between hired service provider and service customers by being a social relator composed by the hired provider commitments and claims and the service customer commitments and claims.

The role of hired service provider is played by an agent $A$, when this agent commits itself to an agent $B$ (playing the role of service customer) to perform actions or to achieve the results determined in the service agreement. This means that service agreement includes a delegation relation [24]: when establishing a service agreement, agent $B$ who plays the role of service customer, delegates a goal/plan to the agent $A$ who plays the role of hired service provider. Thus, claims of $B$ towards $A$, and commitments of $A$ towards $B$ are created, since $A$ has committed to pursue the delegated goal or to execute the delegated plan. Depending on the business service model, this delegation may be open or closed [24]. In open delegation, the hired service provider is free to determine how a commitment is to be fulfilled, which may include further delegation (common in service systems and economic networks). On the other hand, in closed delegation, the hired service provider commits to the execution of a pre-defined plan (i.e., instantiating an agreed action universal).

When agent $B$ delegates a goal/plan to agent $A, B$ becomes (at some level) dependent on $A$. Thus, before hiring a service (and, therefore, establishing a delegation), the customer typically makes an analysis of feasibility, not only associated to monetary aspects, but also to aspects such as dependency, rights, and commitments to be established. Considering the notion of co-responsibility arisen by the mutual commitments, the hired service provider also depends on the service customers for the fulfillment of their own commitments (e.g., a consultancy firm needs access to information from customers in order to provide its services). Thus, in the context of a service agreement, the agent who plays the role of hired service provider $(A)$ is also dependent on the agent who plays the role of service customer $(B)$.

\section{Service Delivery}

Service delivery concerns the execution of actions aimed at fulfilling the commitments established in the service agreement. A service is successfully delivered if the actions are performed in such a way that their results (and also the way they are performed) fulfill the service agreement.

Figure 7 shows an OntoUML class diagram presenting the main concepts and relations involved in service delivery 
according to UFO-S. Service delivery is a complex action, which is composed by several actions, including actions performed only by the hired service provider (hired provider actions), actions performed only by the service customer (customer actions), and actions performed by both in an interaction (hired provider-customer interaction). All of these actions are motivated by the commitments established in service agreement, between the hired provider and the service customer. Depending on the business service model, other agents can also perform actions. For instance, the service provider can delegate some actions to a third-party. These actions are also part of the service delivery process, although they are not explicitly represented in Figure 7.

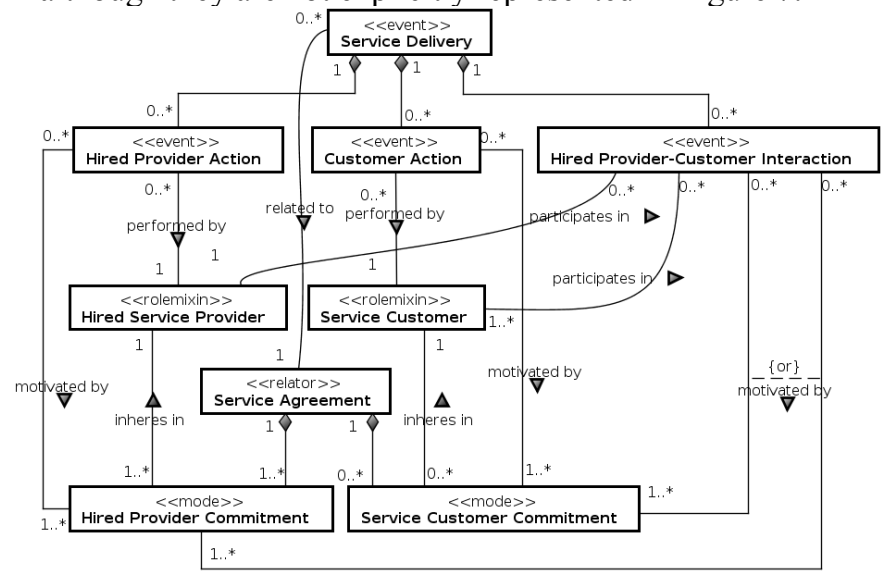

Figure 7. Service Delivery.

\section{APPlying UFO-S to VARIOUS PERSPECTIVES ON SERVICES}

In this section, we evaluate UFO-S by showing how it explains and relates various service perspectives. We cluster these perspectives according to their main characteristics.

\section{A. Service as Co-Creation of Value}

The literature on services often identifies the creation of value as the "raison d'être" for services, i.e., services exist for service participants to benefit or to extract value from their participation. Maglio and Spohrer [4], for example, have characterized service systems as "dynamic configurations of resources capable of providing benefit to other service systems", forming dynamic network structures "(1) capable of improving the state of another system through sharing or applying its resources[...] and (2) capable of improving its own state by acquiring external resources" [3]. Vargo and Lusch have pushed this notion to the extreme, characterizing services as the fundamental basis of value creation through exchange ("all economies are service economies") [2]. Spohrer et al. [3] have explained that "service systems engage in three main activities in order to co-create value: (1) proposing value, (2) accepting a proposal, and (3) realizing the proposal."

In UFO-S, agents become service providers and service customers by participating in intentional actions (service offer, and service negotiation). According to UFO-C, all actions are motivated by an agent's goals and beliefs, even if the result of the actions does not match these goals. Thus, entering into service commitments is motivated by the goals of service providers and service customers, and the beliefs they hold towards service commitments, e.g., that performing a service offer or establishing a service agreement will lead to benefits. However, entering into such commitments cannot be said to guarantee the accomplishment of the motivating goals, e.g., because agents may fail to fulfill their commitments. Even in the case when agents fulfill their commitments, they still may not achieve the goals that led them to enter into such commitments, unless in the trivial case in which the satisfaction of goals is entailed by the fulfillment of commitments.

Whether or not value is produced in the service life-cycle is in fact a subjective notion, which depends on how the service participants assess their participations, i.e., whether they ascribe to the experience in the service life cycle a positive assessment. In this we agree with Vargo and Lusch when they discuss that "value is always uniquely and phenomenologically determined by the beneficiary" and that "value is idiosyncratic, experiential, contextual, and meaning laden." As such, value cannot be (directly) transferred or exchanged. Thus we refrain from using terminology that would suggest otherwise such as "transfers of value" and "service value exchange". This terminology was used initially by Ferrario and Guarino in [14], but then abandoned in [15], when they say that "it is service, not value that is exchanged, because value is subjective". This is not to say that there are no objective outcomes or effects of the actions performed in order to fulfill service commitments. However, the value ascribed to these actions and the situations that are brought about by these actions are subjective.

\section{B. Service as Capabilities and Application of Competences}

Many authors have characterized services by focusing on the capability of providers to produce benefits to customers (e.g., [5][6]) or on the application of such capabilities (competences) of one party in benefit of another party [2].

This is accounted for in UFO-S through the notion of dispositions and their manifestations through events. We regard a capability or competence as a disposition of an agent, be it human or organizational, which under certain conditions is manifested in actions, such as those in the service life-cycle. By combining this explanation with the notion of commitment, UFO-S reveals an important distinction, namely that between: (i) possessing a capability to perform certain actions or to produce certain outcome, i.e., bearing a certain disposition, and (ii) employing capabilities in order to fulfill social commitments, i.e., manifesting the disposition motivated by social commitments. The former is not sufficient for services, since the capability of an organization of washing cars (cf. (i)) does not automatically make it a car wash service provider. Even an organization that is not capable of washing cars may still offer a car wash service, delegating the actual car washing to a party that is capable of doing it. Thus, the capability manifested by the provider is not that of car washing, but that of delegating it to a capable party [36]. In any case, the picture is only complete by considering the commitments that influence the manifestation of dispositions (cf. (ii)). 
Usually in service agreements not only providers but also customers commit themselves to manifesting capabilities under specific conditions. For example, while an online shop commits itself to manifest its capability of shipping goods, the customer commits itself to manifesting his capability of paying for the purchased goods, providing accurate information for delivery, ensuring that someone is present at the delivery address to receive the goods during delivery hours, etc. This view emphasizes the aspect of co-production of services that was discussed in the original ServiceDominant logic article [37] and further shows that the asymmetry in the service provider/service customer relation cannot be explained solely by the application of competences of one party on the behalf of another, because the customer also employs its competences to the benefit of the provider. In order to account for the asymmetry, we need to consider the life-cycle of (meta-)commitments (service offering and service agreement) as discussed in section IV of this article.

\section{Service as Interaction}

The joint participation of service customers and providers has been addressed by some authors who emphasize interactions in the characterization of services. For example, Quartel et al. [1] propose that at a high-level of abstraction, a service can be considered as a single interaction between a "service user" and a "provider". They further propose that at a lower level of abstraction, a service can be regarded as "as multiple related interactions between a service user and provider." This shows that interactions are an important aspect of service delivery. Nevertheless, as we have discussed in the example of the insurance service, it is possible that there is no interaction during service delivery and still the service is provided, due to existence of the service agreement. Further, service delivery includes all actions caused by the intention to fulfill a service agreement, including those referred to in the content of the service agreement in the case of closed delegation. As we discussed in Section IV, some of these actions may involve the participation of service provider and service customer (and are thus interactions), and some may involve only a service provider, or a service customer or a third-party. Depending on the particular service model, service agreements may make reference to both interaction types and particular action types. For example, in the case of the car wash service, the service agreement may include that the car should be vacuum cleaned, specifying thus a type of action to be performed during service delivery. While the view defended in UFO-S encompasses that of service as interaction, we conclude that services should not be reduced only to interactions, and that a broad account must also include the notion of commitments. UFO-S also identifies that interactions occur in other phases of the service lifecycle, e.g., when a service agreement is negotiated.

\section{Technical Services}

At the technical level, the notion of service has been used and defended for over three decades. In [38], for example, service is characterized as observable behavior, in the context of data communication protocols. In that view, a service specification does not reveal any internal structure of the service provider, but it defines the behavior of the provider as it can be observed by users [1][38]. This notion can be explained in UFO-S, since it is possible to establish service agreements with no reference to how the commitments will be fulfilled, by using open delegation.

In a technical service-oriented architecture (such as that discussed in [7]), what counts as a service offer is typically the publication or registration of a service description, or definition of a contract (in the sense of [39]). In this case, the service offering description is often reduced to descriptions of operations or sets of operations that are typically characterized by a pair of interaction types and constraints on them (possibly relating several operations). If operations are considered units of service delivery, then an operation invocation can count as (an implicit and trivial) service negotiation, with the establishment of an agreement whose type is pre-defined in the service offer. In any case, whether service negotiation is implicit, explicit, online or offline, the notion of commitment is instrumental in explaining both the semantics of service description publication and the establishment of Service-Level Agreements (service agreements in UFO-S).

Further, beyond the technical perspective, we believe in line with [16] that service commitments can support us in the relation between the business and the technical perspectives. This is because commitments established between organizational and human agents affects those established at the technical level. By offering an interface through which certain capabilities are leveraged, an operation or a software application should be driven by a set of aspects such as response time, the semantics of data, and possibly the steps followed by the operation / application in order to produce the expected outcome. In contrast, the agent that accesses the operation or the application should respect a set of constraints in order to get the expected result. These aspects can be described in terms of commitments, and can be established at business level, which constrain the implementations as services in operations or applications.

\section{RELATED WORK}

OWL-S [40] and WSMO [41] are Semantic Web Service ontologies that focus mainly on technological aspects and are usually applied for automating tasks such as service discovery and composition [42]. These ontologies are not based on foundational ontologies and none of them addresses the notion of commitments. Further, both are focused on the description of technical services while UFO-S aims instead at explicating service phenomena in order to support (offline) meaning negotiation. Finally, we should note that OWL-S and WSMO are built on semantic web languages that give precedence to computational tractability over expressiveness. As a consequence, these ontologies are less suitable for meaning negotiation.

The Open Group Service-Oriented Architecture Ontology aims to aid understanding in the domain of serviceoriented architecture, and potentially be a basis for modeldriven implementation [37]. In that ontology, the concept of "service" is defined as a logical representation of a 
repeatable activity that has a specified outcome. As we have discussed earlier, this view fails to address the commitment aspects of services. Although the notion of service contract is addressed in this ontology it is considered optional, and the content of contracts is not analyzed in details. No distinction between service offer and service agreement is made. Finally, the Open Group Service-Oriented Architecture Ontology avoids defining concepts such as consumer and provider as core concepts [37], although it mention that these terms may be used in service contracts. This is a consequence of their view on services that, as we have argued in section V.B, cannot account for the asymmetry involved in service provider-customer relations.

The Service Ontology proposed [42] is structured in such way that the core modules span several application domains, such as healthcare, and automotive, and are also grounded by the upper level model, which consists of a concise foundational ontology (DOLCE [21]). By that, we consider this ontology as core reference ontology. The Core Service Description module is one of the most important core modules. It discusses general aspects such as service description, service provider, and service consumer. This module presents two central notions: service and service description. The authors remark that this distinction is important, because in scenarios such as service marketplace the service descriptions are managed instead of the service itself, since a service is defined as an event (in terms of DOLCE). As advocated in UFO-S, the notion of service should be characterized even if no event, interaction, or activity is executed. The notion of commitment as presented in UFO-S as social relators that can be described in service descriptions (including service offering descriptions and service agreement descriptions) may be useful to detail the notion of service description in the Service Ontology.

The Goal-Based Service Ontology (GSO) [18] is a core reference ontology grounded in the same foundational ontology (UFO) of UFO-S. Thus, we can say that both share the same grounding. However, GSO focuses on the concept of goal in order to discuss the notion of service. In GSO, service is as a temporal entity related to the commitment that a service provider will perform a task on behalf of a service client whose outcome satisfies a service client's goal [18]. The notion of commitments addressed in GSO lacks further discussions. For example, GSO does not discuss the kinds of commitments and how these kinds of commitments influence the definition of the agents' roles along the service life-cycle. As a consequence, in GSO, it is not possible to discuss the notion of target service customer, and hired service provider, for example. Also, in the service definition of GSO, only the provider is committed to achieving the goal. However, service relationships are usually characterized by mutual commitments, since the service client is also committed.

Ferrario and Guarino in [14][15] present an ontological analysis of service systems that is also based on the notion of commitments. The most salient differences between UFO-S and such previous works are: (i) the adoption of OntoUML, and (ii) the grounding in UFO (instead of DOLCE). With respect to the former, OntoUML offers well-founded modeling capabilities that are associated to a number of tools (e.g., model verification and simulation [43] and generation of OWL implementations [44]). Regarding the latter, we highlight the use of the social and intentional distinctions underlying UFO-C, and that, besides offering a hierarchy of individuals, UFO (in contrast to DOLCE) also offers a hierarchy of universals, which contributes, among others, to the definition of types required in our account (e.g. role mixins, relator universals). Further work is however necessary to investigate the relationship between UFO relators and DOLCE states.

\section{FINAL CONSIDERATIONS}

This work presented the definition of a foundation for Service Computing and Service Science with a core reference ontology for services called UFO-S. UFO-S is grounded in a foundational ontology that includes social and intentional concepts that form the basis for our account of the social relations established throughout the service lifecycle. UFO-S accounts for the agent's intentionality with respect to the actions they perform by entering into and aiming to fulfill their social commitments.

We have shown that UFO-S is able to explain a number of perspectives on services, including those that emphasize services as value co-creation, services as capabilities, services as application of competences, services as interactions and, finally, services as a technical notion.

In order to relate the various perspectives, we have refrained from settling on a specific definition for the term "service", understanding that the term is laden with different meanings (e.g., differently from the approach taken in [14][15]). Our approach addresses the elements of the phenomena underlying services, providing a foundation that can be used to articulate the semantics of service-related terminology. With that we hope not to incite the terminological debate, which in our opinion should not play a dominant role in the development of solid foundations for Service Science and Services Computing.

We have argued that the notion of service commitments can span the business and technical perspectives, with the potential of promoting a much-needed conceptual integration of business and IT service aspects. We believe this observation can guide our future work on well-founded modeling of service-oriented enterprise architectures. Thus, we intend to perform an ontological analysis of ArchiMate focusing on the service construct. This would contribute to the agenda we have been pursuing in the last decade towards well-founded enterprise modeling [26][27] [28][29].

We have focused on a core fragment of the service life cycle, leaving marketing-related phases (pre-service offer and post-service delivery) outside the scope of this paper. Future work could expand on that in order to provide a more detailed account for the origin of value propositions and to further explicate the subjective value experience aspects. Also is necessary to account for issues regarding resource allocation, usage and consumption in service delivery.

Our use of the well-founded OntoUML profile for modeling UFO-S leverages the conceptual distinctions in UFO as well as the tool support already developed for OntoUML; This includes formal verification of the model 
(guaranteeing that the models put forth here are compliant with the UFO ontological constraints), validation of the model via visual simulation (relying on an OntoUML infrastructure developed on top of the Alloy Analyzer [43]) as well as the systematic implementation of UFO-S in computational level languages such as OWL [44].

\section{ACKNOWLEDGMENTS}

This research is funded by the Brazilian Research Agencies CAPES/CNPq (402991/2012-5), FAPES/CNPq (PRONEX $52272362 / 11)$, FAPES (59971509/12) and $\mathrm{CNPq}$ (483383/2010-4, 310634/2011-3, and 311578/2011-0).

\section{REFERENCES}

[1] D. A. C. Quartel, M. W. A. Steen, S. Pokraev, and M. J. van Sinderen, "COSMO: A conceptual framework for service modelling and refinement," Information Systems Frontiers, vol. 9, no. 2-3, pp. 225244, 2007.

[2] S. L. Vargo and R. L. Lusch, "Evolving to a new dominant logic for marketing," Journalof Marketing, vol. 68, pp. 1-17, 2004.

[3] J. Spohrer, S. L. Vargo, N. Caswell, and P. P. Maglio, "The service system is the basic abstraction of service science," in 4lst Annual Hawaii International Conference on System Sciences HICSS, 2008, vol. 7, pp. 104-104.

[4] P. P. Maglio and J. Spohrer, "Fundamentals of Service Science," Journal of the Academy of Marketing Science, vol. 36, no. 1, pp. 18$20,2008$.

[5] "Reference Model for Service Oriented Architecture 1.0: OASIS Standard." OASIS, pp. 1-31, 2006.

[6] T. Ruokolainen, "A Model-Driven Approach to Service Ecosystem Engineering," University of Helsinki, 2013.

[7] W3C, "Web services architecture W3C: Working Group Note 11," 2004. [Online]. Available: http://www.w3.org/TR/ws-arch/.

[8] "Archimate 2.0 Specification: Technical Standard." The Open Group, Berkshire, UK, 2012.

[9] S. Alter, "Service System Fundamentals: Work System, Value Chain, and Life Cycle," IBM Systems Journal, vol. 47.1, pp. 71-85, 2008.

[10] D. Oberle, Semantic Management of Middleware. Berlin: SpringerVerlag, 2006.

[11] A. Scherp, C. Saathoff, T. Franz, and S. Staab, "Designing core ontologies," Applied Ontology, vol. 6, no. 3, pp. 177-221, 2011.

[12] G. Guizzardi, "Ontological Foundations for Structural Conceptual Models," University of Twente, 2005.

[13] G. Guizzardi, R. A. Falbo, and R. S. S. Guizzardi, "Grounding software domain ontologies in the Unified Foundational Ontology (UFO): the case of the ODE software process ontology," in Proceedings of the XI Iberoamerican Workshop on Requirements Engineering and Software Environments, 2008, pp. 244-251.

[14] R. Ferrario and N. Guarino, "Towards an Ontological Foundation for Services Science," in First Future Internet Symposium (FIS), 2008, vol. Vol. 5468, pp. 152-169.

[15] R. Ferrario and N. Guarino, "Commitment-based Modeling of Service Systems," in Third International Conference, IESS, 2012, pp.170-185.

[16] M. P. Singh, A. K. Chopra, and N. Desai, "Commitment-Based Service-Oriented Architecture" Computer, vol. 42, no. 11, pp. 72-79, 2009.

[17] W. Mingming and H. Youbei, "Design and Implementation of Service Commitment," in International Conference on Service Sciences ICSS, 2010, pp. 388-391.

[18] L. O. B. S. Santos, G. Guizzardi, R. S. S. Guizzardi, E. G. da Silva, L. F. Pires, and M. Van Sinderen, "GSO: Designing a Well-Founded Service Ontology to Support Dynamic Service Discovery and Composition," in 13th Enterprise Distributed Object Computing Conference Workshops, 2009, pp. 35-44.

[19] J. O'Sullivan, "Towards a Precise Understanding of Service Properties," Queensland University of Technology, 2006.

[20] M. Dumas, J. O’Sullivan, M. Hervizadeh, D. Edmond, and A. H. M. Ter Hofstede, "Towards a Semantic Framework for Service Description," in IFIP TC2WG26 Ninth Working Conference on
Database Semantics Semantic Issues in ECommerce Systems, 2001, pp. 277-291.

[21] O. A. Masolo C., Borgo S., Gangemi A., Guarino N., "WonderWeb DeliverableD18. Ontology Library," Padova-Italy, 2003.

[22] N. Guarino and C. Welty, "Evaluating ontological decisions with OntoClean," Communications of the ACM, v. 45, no. 2,p. 61-65, 2002.

[23] B. Heller and H. Herre, "Ontological Categories in GOL" Axiomathes, Kluwer Academic Publishers, vol. 14, no. 1, pp. 57-76, 2004.

[24] R. S. S. Guizzardi, "Agent-Oriented Constructivist Knowledge Management," University of Twente, 2006.

[25] A. C. O. Bringuente, R. A. Falbo, and G. Guizzardi, "Using a Foundational Ontology for Reengineering a Software Process Ontology," Journal of Information and Data Management, vol. 2, pp. 511-526, 2011.

[26] P. S. Santos Jr., J. P. A. Almeida, and G. Guizzardi, "An ontologybased analysis and semantics for organizational structure modeling in the ARIS method," Information Systems (Oxford), pp. 690-708, 2012.

[27] C. Azevedo, J. P. A. Almeida, M. J. Van Sinderen, D. Quartel, and G. Guizzardi, "An Ontology-Based Semantics for the Motivation Extension to ArchiMate," in 15th IEEE International Conference on Enterprise Computing (EDOC), 2011, Helsinki, Finland., pp. 25-34.

[28] J. P. A. Almeida and G. Guizzardi, "An Ontological Analysis of the Notion of Community in the RM-ODP Enterprise Language," Computer Standards \& Interfaces, vol. 34, pp. 1, 2012.

[29] J. P. A. Almeida, G. Guizzardi, and P. S. Santos Jr., "Applying and Extending a Semantic Foundation for Role-Related Concepts in Enterprise Modelling," Enterprise Information Systems (Print), vol. 3 pp. 253-277, 2009

[30] J. Heil, From an Ontological Point of View. Oxford University Press, USA, 2005

[31] S. Mumford, Dispositions. Oxford University Press, 2003.

[32] G. Guizzardi, "Agents Roles, Qua Individuals and The Counting Problem," Software Engineering of Multi-Agent Systems, vol. 4, pp. $143-160,2005$

[33] R. Ferrario and A. Oltramari, "Towards a Computational Ontology of the Mind," in 3rd International Conference on Formal Ontology in Information System (FOIS), 2004

[34] J. R. Searle, Mind, Language and Society: Philosophy In The Real World. Basic Books, 2000, p. 626.

[35] C. Castelfranchi, "Commitments: From Individual Intentions to Groups and Organizations," in Proceedings of the First International Conference on Multiagent Systems, 1995, pp. 41-48.

[36] R. S. S. Guizzardi and G. Guizzardi, "Ontology-Based Transformation Framework from Tropos to AORML," In Social Modeling for Requirements Engineering. Cooperative Information Systems Series, P. Giorgini, N. Maiden, J. Mylopoulos, and E. Yu, Eds. Boston: MIT Press., 2010.

[37] "Service-Oriented Architecture Ontology." The Open Group, 2009.

[38] C. Vissers and L. Logrippo, "The importance of the service concept in the design of data communication protocols", Protocol Specification, Testing and Verification, vol. V, pp. 3-17, 1986.

[39] B. Meyer, "Applying 'Design by Contract'”, Computer, vol. 25, no. 10, pp. 40-51, 1992.

[40] D. Martin, M. Burstein, J. H. O. Lassila, D. McDermott, and E. Al., "OWL-S: Semantic markup for web services: W3C Member Submission 22.," $2004 . \quad$ [Online]. Available: http://www.w3.org/Submission/OWL-S/.

[41] J. de Bruijn, C. Bussler, J. Domingue, D. Fensel, M. Hepp, M. Kifer, B. König-Ries, J. Kopecky, R. Lara, E. Oren, A. Polleres, J. SciCluna and M. Stollberg, "Web Service Modeling Ontol ogy (WSMO). Final Draft.," 2006. [Online]. Available: http://www.wsmo.org/TR/d2/v1.3/.

[42] D. Oberle, N. Bhatti, S. Brockmans, and C. Janiesch, "Countering service information challenges in the internet of services.," Journal of Business \& Information System Engineering (BISE), vol. 5, 2009.

[43] A. B. Benevides, G. Guizzardi, B. F. B. Braga, and J. P. A. . Almeida "Validating Modal Aspects of OntoUML Conceptual Models Using Automatically Generated Visual World Structures," Journal of Universal Computer Science (Print), vol. 16, pp. 2904-2933, 2011.

[44] V. Zamborlini and G. Guizzardi, "On the Representation of Temporally Changing Information in OWL," in Enterprise Distributed Object Computing Conference Workshops (EDOCW) - VORTE, 2010, pp.283-292. 